\title{
Construcción de Bipedestor para rehabilitación y caminata para niños parapléjicos de 2 a 3 años
}

\section{Construction of Bipedestor for rehabilitation and walking for paraplegic children from 2 to 3 years old}

\section{Eduardo Francisco García-Cabezas}

egarcia@espoch.edu.ec

Escuela Superior Politécnica de Chimborazo. Grupo de Investigación \& Vinculación

AUTOPRO, Riobamba

Ecuador

https://orcid.org/0000-0002-3547-472X

Kevin Humberto Álvarez-Núñez

kevinkhan1994@gmail.com

Investigador Independiente

Ecuador

https://orcid.org/0000-0003-1367-8235

Carlos José Santillán-Mariño

carlos.santillan@espoch.edu.ec

Escuela Superior Politécnica de Chimborazo. Grupo de Investigación \& Vinculación

AUTOPRO, Riobamba

Ecuador

https://orcid.org/0000-0002-7167-7452

Juan Carlos Cayán-Martínez

jcayan@espoch.edu.ec

Escuela Superior Politécnica de Chimborazo. Grupo de Investigación \& Vinculación

AUTOPRO, Riobamba

Ecuador

https://orcid.org/0000-0001-9573-3706

Recibido: 10 de marzo de 2021

Aprobado: 15 de junio de 2021 


\title{
RESUMEN
}

El objetivo general de la investigación es construir un bipedestor para rehabilitación y estimulación de caminata pasiva para niños parapléjicos de 2 a 3 años. El diseño del bipedestor fue basado en medidas antropométricas promedios de niños en el Ecuador de 2 a 3 años de edad, para la selección del material se ha efectuado un análisis estático en el software Solidworks. Posee un arnés de seguridad para sostener al niño cubierto con esponjas tapizadas para evitar algún tipo de golpe o impacto previniendo accidentes. Para el sistema de automatización cuenta con una interfaz gráfica donde muestra los datos del paciente y los pulsadores de encendido y apagado del cilindro neumático. Se concluye que se logró desarrollar un bipedestor, diseñando e implementando un diseño mecánico automatizado, recomendando utilizar el arnés de seguridad acoplado en las piernas cada vez que se inicie la rehabilitación y disponer del acompañamiento de personas adultas.

Descriptores: Diseño; rehabilitación; interfaz de ordenadores. (Palabras del Tesauro UNESCO).

\begin{abstract}
The general objective of the research is to build a walking stick for rehabilitation and passive walking stimulation for paraplegic children from 2 to 3 years old. The bipedestor design was based on average anthropometric measurements of children in Ecuador between 2 and 3 years of age. For the selection of the material, a static analysis was carried out in the Solidworks software. It has a safety harness to hold the child covered with upholstered sponges to avoid any type of blow or impact, preventing accidents. For the automation system it has a graphical interface where it shows the patient data and the pneumatic cylinder on and off buttons. It is concluded that it was possible to develop a standing post, designing and implementing an automated mechanical design, recommending using the safety harness attached to the legs each time rehabilitation begins and having the accompaniment of adults.
\end{abstract}

Descriptors: Design; rehabilitation; computer interface. (Words from UNESCO Thesaurus). 


\section{INTRODUCCIÓN}

Según la organización mundial de la salud estima que el 15\% de la población mundial son afectados por alguna discapacidad física o psicológica. En Ecuador el 5.64\% de personas poseen discapacidades según la CONADIS, dificultando su interacción independiente en la sociedad. La mayoría de personas necesitan rehabilitaciones diarias y no lo hacen debido a la falta de tiempo o económicamente no lo pueden sustentar. (Sáenz \& Chacón, 2014, pp. 18-19)

Constantemente en el país trabajan en leyes para mejorar la calidad de vida de personas con discapacidades, y con la ayuda de la tecnología se ha implementado nuevas herramientas que han logrado desarrollar prótesis de extremidades superiores e inferiores accesibles para la población, debido que son realizadas mediante impresoras 3D, generando un bajo costo en material, incluso se ha innovado y muchas prótesis son automatizadas. (Sáenz \& Chacón, 2014, p. 18)

Se ha considerado que gran parte de personas con paraplejia necesitan rehabilitaciones diarias, al menos en niños necesitan aprovechar los 5 primeros años para fortalecer la estructura de los miembros inferiores, aparte de las sesiones de fisioterapia que poseen necesitan pasar en posición bípeda viarios minutos al día que sería parte de su rehabilitación y provocando un mejor funcionamiento de órganos internos del cuerpo humano.

El trabajo de titulación se enfoca en la construcción de un bipedestor para un niño de 2 años brindando la facilidad de realizar una rehabilitación desde su casa u otro lugar que no sea el centro de fisioterapia con el motivo de estar constantemente de pie, la máquina construida aporta con una sistema de control automatizado permitiendo la movilidad del bipedestor y siendo totalmente independiente, cuenta con un actuador que está regulado para realizar dos movimientos de sedestación y bipedestación en un tiempo determinado de esta manera se incluye otro método de rehabilitación en la máquina, esto es controlado por un sistema de software libre una raspberry pi 3 Model $\mathrm{B}+$ que por medio de un HDMl que muestra la interfaz gráfica para el paciente. 


\section{Rehabilitación robótica}

La rehabilitación con asistencia de tecnologías para personas con carencias en sus extremidades motoras es una posibilidad de tratamiento funcional para recuperaciones físicas con diseños basados en estudios médicos, muestra una mejora en su rendimiento y los pacientes realizan diferentes modalidades de ejercicios terapéuticos que restauran sus movimientos, optimizando el control de movilidad de su cuerpo con ayuda de máquinas electromecánicas, obteniendo una mejor recuperación. De esta manera los pacientes pueden llevar una vida autónoma y mejorar su calidad de vida. (Criollo \& David, 2011, pp. 60-61) (Meneses \& Peñaloza, 2015).

\section{Interfaz hombre máquina}

En la mayoría de metodologías industriales tiene la necesidad de aplicar la automatización en controles de métodos para los diferentes campos de producción, enfocándose especialmente en la calidad del resultado final, para esto es necesario tener en cuenta la ejecución de técnicas nuevas acordes a las necesidades de los usuarios sacando el mayor provecho a los sistemas informáticos. Es ideal la comunicación estable entre máquinas y operarios partiendo de un sistema de control automatizado, con esta finalidad desarrolla interfaces para usuarios con técnicas de análisis de actividades. (Ruiz \& Inche, 2008, pp. 70-72)

\section{Definición interfaz hombre máquina}

Se refiere a una interacción con nuevas tecnologías mostrando una comunicación entre elementos gráficos y los usuarios, es decir, desarrollando una interfaz entre operadores y máquinas analizando cuidadosamente variables de información, proporción de herramientas y técnicas para cubrir los objetivos propuestos para los usuarios. Para el desarrollo de la interfaz es recomendable utilizar términos estandarizados como componentes numéricos y componentes gráficos, de esta forma 
mantener registros de las variaciones ocurridas para el estudio del comportamiento de las mismas. (Ruiz \& Inche, 2008, p. 72)

\section{Bipedestación}

La bipedestación esta defina como la habilidad del cuerpo humano para mantenerse en pie, debido a la alineación de los músculos que mantienen el cuerpo en equilibrio como el cuello y el tronco, el peso se encuentra distribuido en la base de los pies y de esta manera su centro de gravedad está equidistante. Es necesario mantener esta posición para el correcto desplazamiento del cuerpo humano. (Luengas \& Esperanza, 2013, pp. 61-63)

\section{Beneficios de la bipedestación}

Mantener la posición bípeda produce sensaciones buenas para la salud como mayor energía y bienestar, ayudando a prevenir dolores y contracturas de extremidades inferiores, beneficiando especialmente al sistema circulatorio evitando que se produzcan edemas, reduciendo la formación de úlceras por presión. Otro beneficio es la ayuda que presenta al crecimiento correcto de los huesos. Previniendo la osteoporosis y disminuyendo fracturas, siendo el desarrollo para poder levantarse independientemente y mantener una posición erguida. (Bermeo, 2012, p. 13)

\section{MATERIALES Y METODOS METODOLOGÍA}

El presente trabajo de investigación tiene como objetivo la implementación de Ingeniería Industrial y recursos tecnológicos Open Source disponibles, para equipos de rehabilitación para personas con capacidades diferentes. Pretendiendo presentar alternativas genéricas a un menor costo accesible para la ciudadanía y brindando ayuda social a la misma. Según el autor presenta una metodología propia que es descrita por medio de un diagrama de bloques en la figura 18-2. 


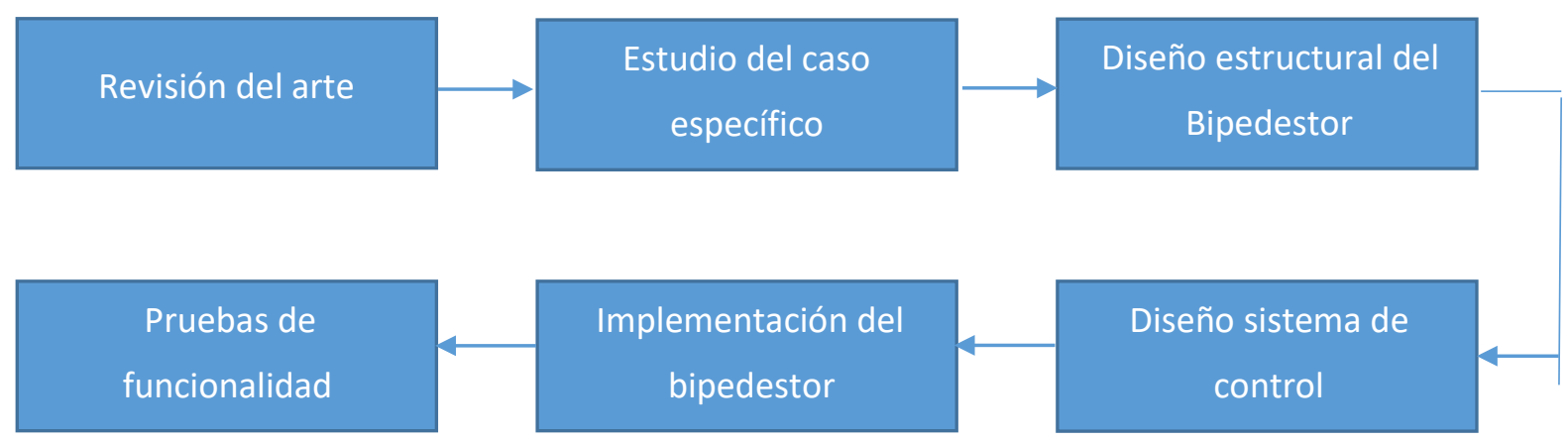

Figura 1. Metodología.

\section{Fuente: Los autores.}

\section{Estudio del caso específico}

El estudio del caso específico es dirigido especialmente a un niño de dos años y medio de edad de la ciudad de Riobamba-Ecuador, quien obtuvo un trauma obstétrico que se define como las lesiones que presentan los recién nacidos en labor de parto por las maniobras y manipulaciones generalmente al pasar por el canal del parto por tracción y presión ejercidas. Estos traumas dejan lesiones graves o incluso pueden conducir a la muerte, las lesiones comunes puede ser (heridas, erosiones, contusiones, lesiones craneales, problemas en el funcionamiento de la vejiga). (Murguía \& Hernández, 2013, pp. 298-299)

El trauma obstétrico provoco una fractura bilateral de fémur y lesión lumbosacra por lo tanto, el niño no posee movilidad en los miembros inferiores e incluso le realizaban estímulos dolorosos y no había respuesta, además, posee en el miembro superior derecho poca movilidad y mantiene puño cerrado. (Espinoza, 2003, pp. 322-323).El niño recibe rehabilitaciones tres días por semana, ha demostrado avances en la movilidad de sus extremidades, terapeutas recomiendan un bipedestor para el mejor funcionamiento de sus aparatos digestivos y respiratorios, circulación de la sangre y otros beneficios que presenta un bipedestor. (Saavedra \& García, 2013, p. 26) 


\section{Proceso de rehabilitación}

Es un proceso que tiene como objetivo compensar las consecuencias de una enfermedad haciendo uso de medidas físicas médicas y educativas, ayudando a personas con capacidades diferentes en sus limitaciones físicas alcanzando posibles mejores y adaptaciones al entorno social. (Galindo \& Gloria, 1997)

\section{Rehabilitación integral}

Proceso por el cual el paciente entiende las desventajas al que está expuesto por su capacidad diferente, comprendiendo las áreas de rehabilitación funcional, profesional y social.

La persona debe tener el conocimiento del desempeño que puede cumplir en ciertas funciones y la voluntad de trabajo que debe presentar.

El medio social disponga de oportunidades de acuerdo a las capacidades de las personas, disminuyendo barreras para la integración con la sociedad. (Galindo \& Gloria, 1997)

\section{Rehabilitación profesional}

Incluir a personas con capacidades diferentes a trabajos acordes a sus habilidades y destrezas.

Facilitar la capacitación para el puesto de trabajo.

Promover la seguridad del trabajo. (Galindo \& Gloria, 1997)

\section{Rehabilitación con bipedestación}

Trata de una rehabilitación que puede ejercer en un gimnasio con la dirección de fisioterapistas con distintos ejercicios en barras paralelas, la bipedestación es un tipo de rehabilitación que debe realizarse de forma inmediata por sus beneficios enfocándose en un entrenamiento denominado "sit to stand" mostrando ventajas como extenso movimiento de todas articulaciones de los miembros inferiores, activación de 
sistemas circulatorio y respiratorio, prevención de múltiples enfermedades. (Catañeda, 2014)

\section{Bipedestor propuesto}

El trabajo de investigación en forma general desea cumplir con la construcción e implementación de un bipedestor que cuente con la facilidad de trasladarse a varios lugares, debido que posee motores que generan el movimiento que son controlados por el paciente. Además, cuenta con un sistema de rehabilitación con un cilindro neumático que permite el movimiento de sus miembros inferiores permitiendo que el paciente sea autónomo con la posibilidad de mantenerse de pie varias veces al día, el bipedestor le brinda ayuda para el desarrollo de actividades propias de niños de su edad, su rehabilitación sería constante logrando una contribución psicológica para el paciente y a la vez para la sociedad. (Quiñones \& Robayo, 2013, p. 424)

Los bipedestadores que se encuentran generalmente en hospitales y centros de rehabilitación son de madera o estructura metálica, son rígidos y no cuentan con una rehabilitación completa solo se ocupan de mantener al paciente en una posición bípeda. Por lo tanto, el trabajo de titulación propone una rehabilitación para sus miembros inferiores. (Saavedra \& García, 2013) 


\section{CIENCIAMATRIA}

Revista Interdisciplinaria de Humanidades, Educación, Ciencia y Tecnología Año VII. Vol. VII. N¹. Edición Especial. 2021 Hecho el depósito de ley: pp201602FA4721

ISSN-L: 2542-3029; ISSN: 2610-802X

Universidad Nacional Experimental Francisco de Miranda (UNEFM). Santa Ana de Coro. Venezuela

Eduardo Francisco García-Cabezas; Kevin Humberto Álvarez-Núñez; Jhonny Marcelo Orozco-Ramos; Juan Carlos Cayán Martínez

\section{RESULTADOS}

\section{Construcción del bipedestor}

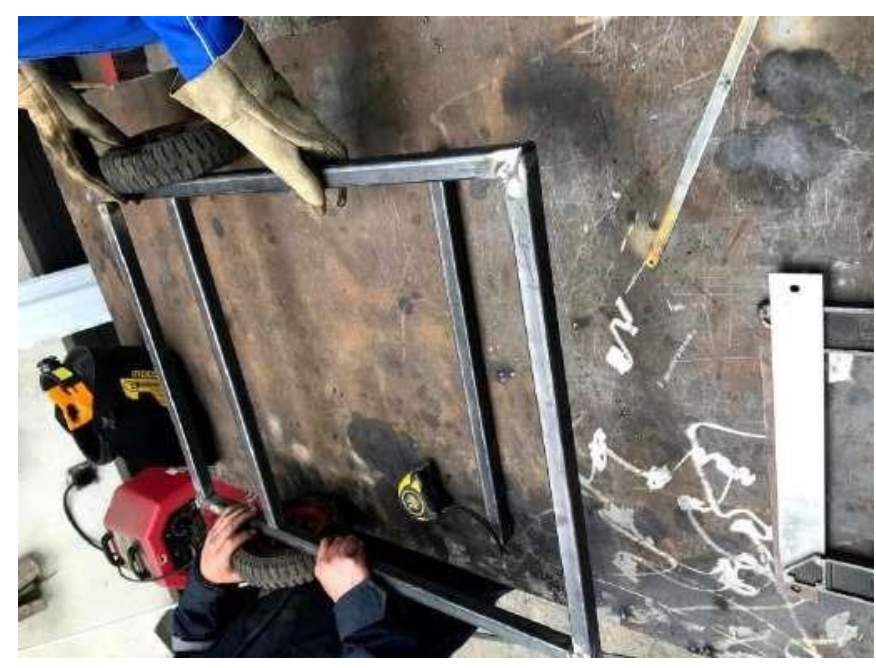

Figura 2. Construcción de la estructura base.

Fuente: Los autores.

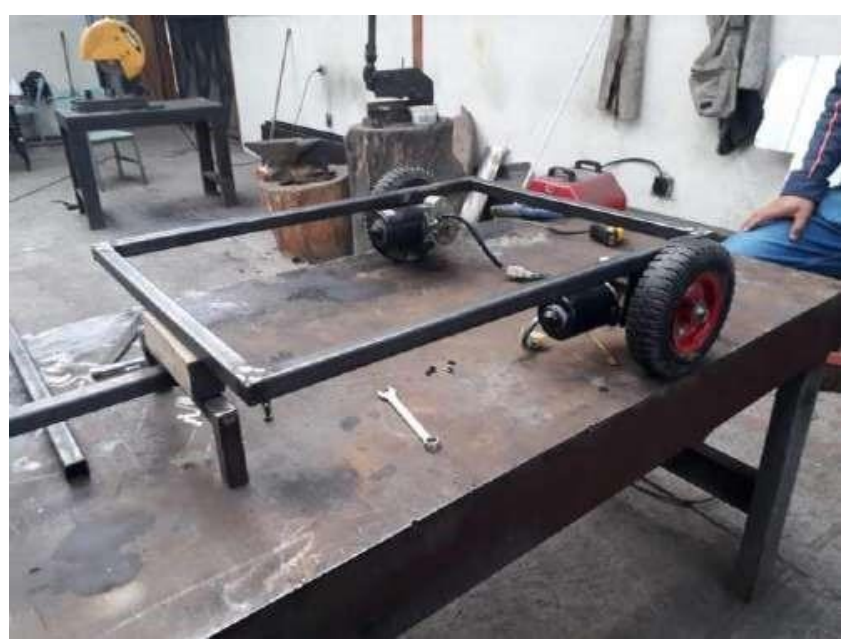

Figura 3. Ensamble de motores.

Fuente: Los autores. 


\section{CIENCIAMATRIA}

Revista Interdisciplinaria de Humanidades, Educación, Ciencia y Tecnología

Año VII. Vol. VII. N¹. Edición Especial. 2021

Hecho el depósito de ley: pp201602FA4721

ISSN-L: 2542-3029; ISSN: 2610-802X

Universidad Nacional Experimental Francisco de Miranda (UNEFM). Santa Ana de Coro. Venezuela

Eduardo Francisco García-Cabezas; Kevin Humberto Álvarez-Núñez; Jhonny Marcelo Orozco-Ramos; Juan Carlos Cayán Martínez

Para la construcción física del Bipedestor en primera instancia se procede armar la estructura base según las medidas establecidas y los materiales seleccionados en el diseño realizado en SolidWorks, seguido se ensambla las ruedas traseras en las cuales son acoplados los motores que generan el movimiento a todo el cuerpo y las ruedas delanteras que no tienen dirección debido a que el giro del bipedestor es controlado por las ruedas traseras.

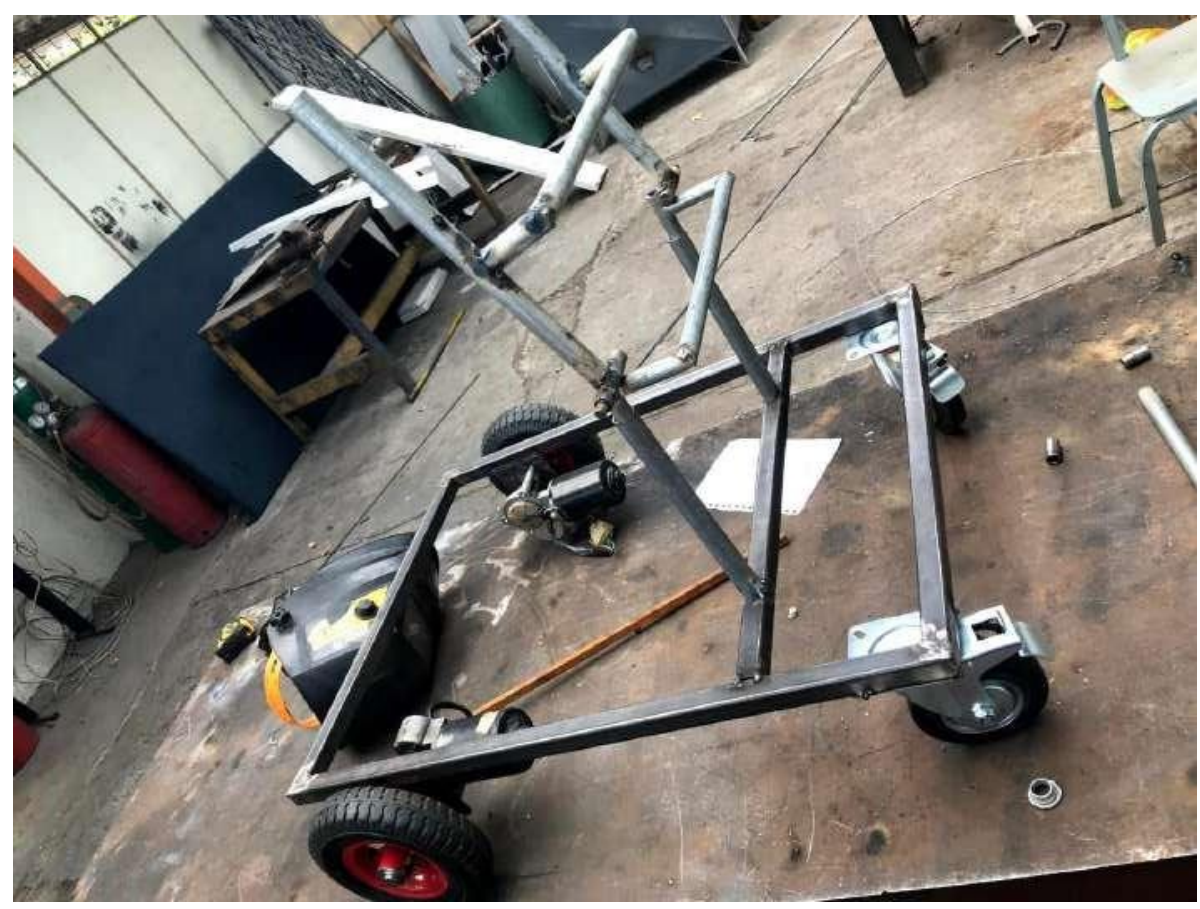

Figura 4. Ensamble de los soportes. Fuente: Los autores.

En la figura 4. Es ensamblado los soportes fijo y móvil que generan el movimiento para la rehabilitación y estimulación a la caminata pasiva del niño. Los soportes son construidos de acuerdo a los materiales seleccionados para esto se realizó pruebas y medidas con el niño beneficiado de la máquina de rehabilitación. 
Revista Interdisciplinaria de Humanidades, Educación, Ciencia y Tecnología

Año VII. Vol. VII. N¹. Edición Especial. 2021

Hecho el depósito de ley: pp201602FA4721

ISSN-L: 2542-3029; ISSN: 2610-802X

Universidad Nacional Experimental Francisco de Miranda (UNEFM). Santa Ana de Coro. Venezuela

Eduardo Francisco García-Cabezas; Kevin Humberto Álvarez-Núñez; Jhonny Marcelo Orozco-Ramos; Juan Carlos Cayán Martínez

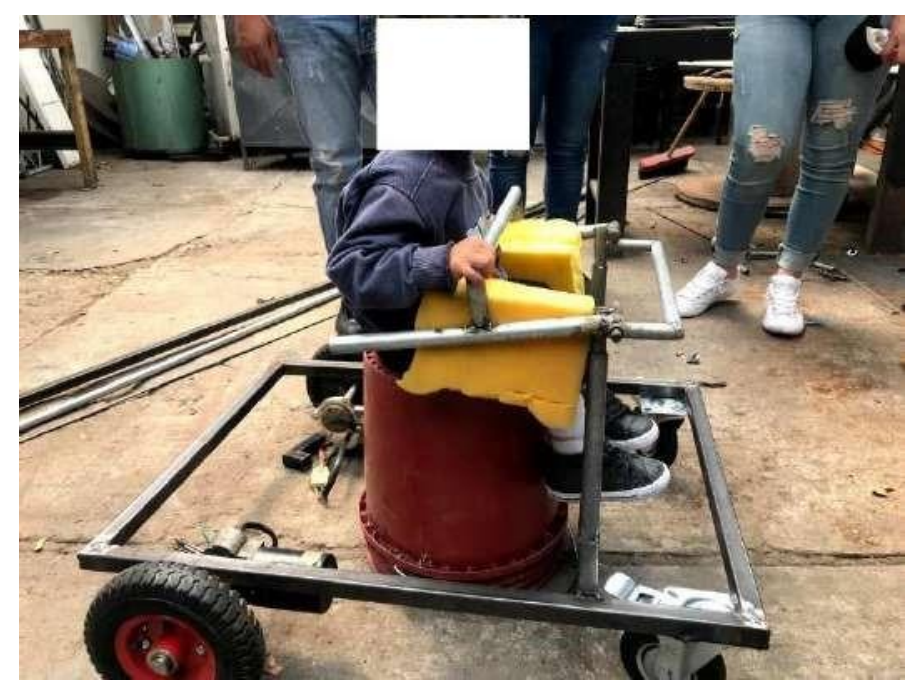

Figura 5. Pruebas de medidas con el niño.

Fuente: Los autores.

En la figura 5. Se observa que la construcción de la estructura del bipedestor cumple con las medidas requeridas para la estatura del niño, en esta parte se tomó las medidas exactas de los ángulos para que el niño se encuentre en las posiciones de bipedestación y sedestación. Por lo tanto, se colocó topes estratégicos para evitar cualquier tipo de accidente, es decir, el bipedestor no sobrepasara los ángulos establecidos para la rehabilitación física. 
Revista Interdisciplinaria de Humanidades, Educación, Ciencia y Tecnología

Año VII. Vol. VII. N¹. Edición Especial. 2021

Hecho el depósito de ley: pp201602FA4721

ISSN-L: 2542-3029; ISSN: 2610-802X

Universidad Nacional Experimental Francisco de Miranda (UNEFM). Santa Ana de Coro. Venezuela

Eduardo Francisco García-Cabezas; Kevin Humberto Álvarez-Núñez; Jhonny Marcelo Orozco-Ramos; Juan Carlos Cayán Martínez

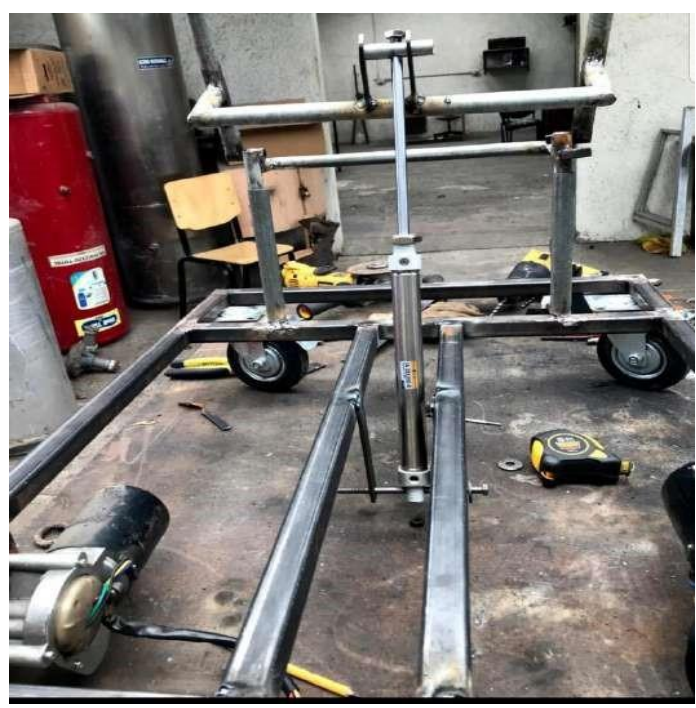

Figura 6. Ensamble del cilindro neumático doble efecto. Fuente: Los autores.

En la figura 6. Se ensambla el cilindro neumático que se extiende $25 \mathrm{~mm}$ que es lo ideal para cumplir con el recorrido para ejercer la rehabilitación, cabe mencionar que en las bases del cilindro, soporte y mesa de apoyo existen regulaciones con medidas de separaciones de 3 centímetros para que el niño puedo ocupar la máquina mientras vaya cambiando de estatura. 


\section{CIENCIAMATRIA}

Revista Interdisciplinaria de Humanidades, Educación, Ciencia y Tecnología Año VII. Vol. VII. N¹. Edición Especial. 2021 Hecho el depósito de ley: pp201602FA4721

ISSN-L: 2542-3029; ISSN: 2610-802X

Universidad Nacional Experimental Francisco de Miranda (UNEFM). Santa Ana de Coro. Venezuela

Eduardo Francisco García-Cabezas; Kevin Humberto Álvarez-Núñez; Jhonny Marcelo Orozco-Ramos; Juan Carlos Cayán Martínez

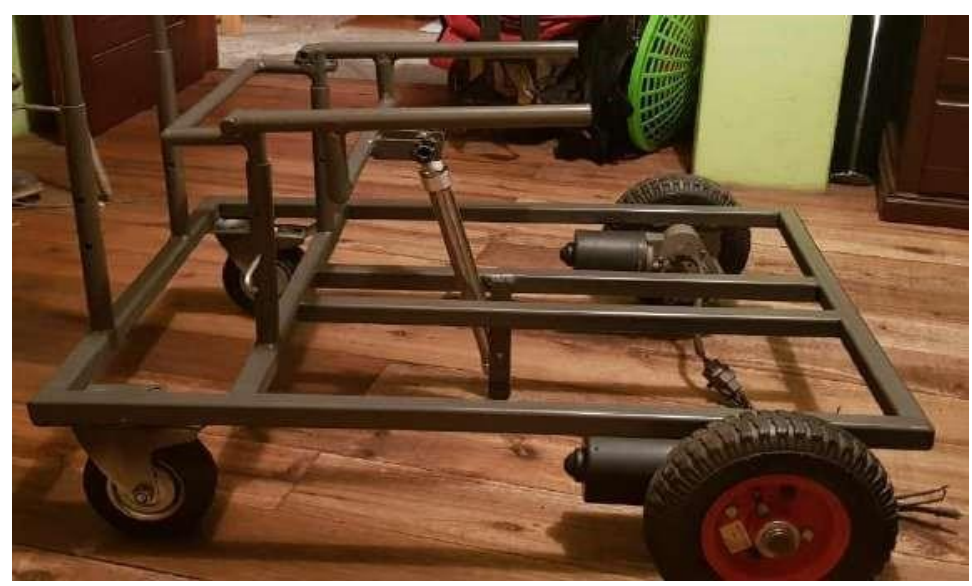

Figura 7. Ensamblaje final.

Fuente: Los autores.

Finalmente en la figura 7. Muestra el ensamble final de la estructura presto a colocar el piso y la implementación de los circuitos neumáticos, eléctricos y electrónicos.

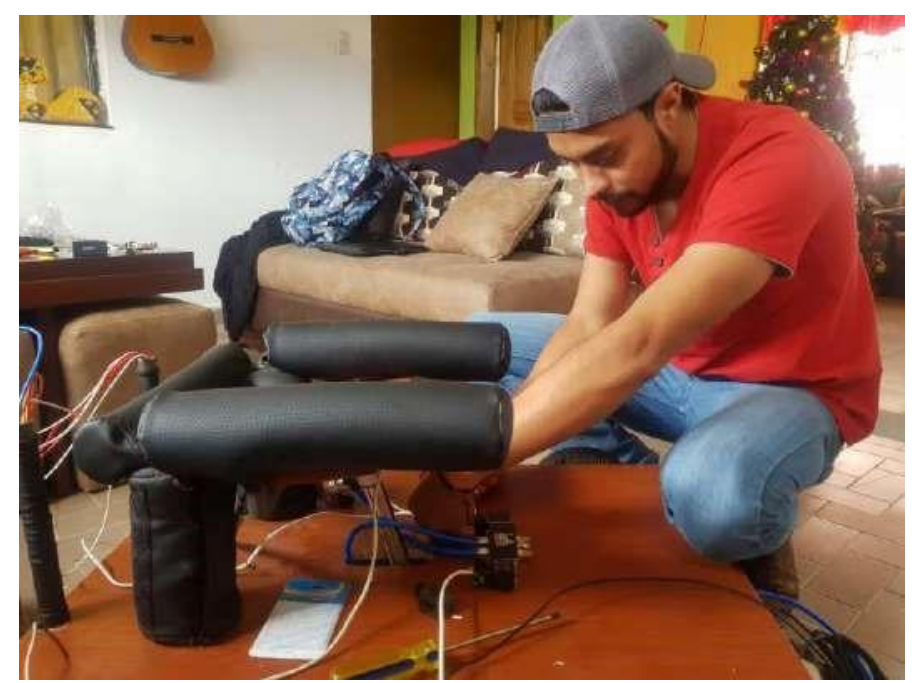

Figura 8. Ensamble circuitos neumáticos.

Fuente: Los autores. 
En la figura 8. Muestra el ensamble de la electroválvula y las conexiones neumáticas instaladas abajo del piso para evitar que el niño tenga algún contacto con las mangueras del cilindro, cuidando la integridad física del niño y el funcionamiento del bipedestor.

\section{Montaje de circuitos electrónicos}

Una vez programados los elementos de control para el bipedestor se procede a realizar las respectivas conexiones colocando en orden los dispositivos a ser utilizados, la máquina de rehabilitación es para niños está expuesta a daños involuntarios o accidentes con los circuitos, por esta razón se ha decido colocar los elementos dentro de la mesa que forma una caja cerrada sin acceso para el usuario, solo para el programador como se muestra en la figura 9.

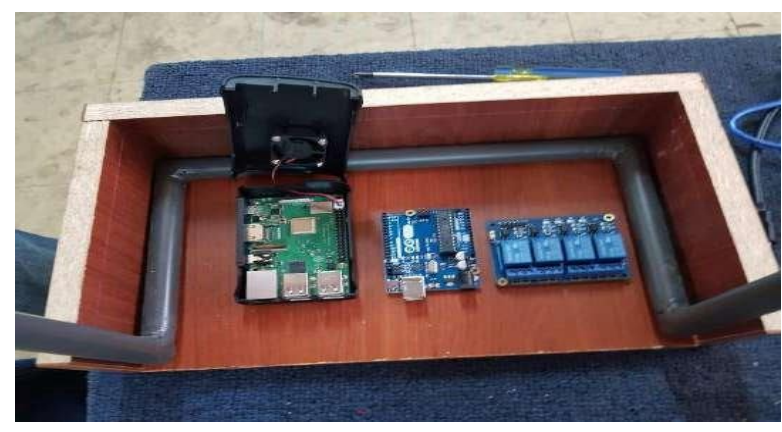

Figura 9. Montaje de los dispositivos electrónicos. Fuente: Los autores. 
Revista Interdisciplinaria de Humanidades, Educación, Ciencia y Tecnología

Año VII. Vol. VII. N¹. Edición Especial. 2021

Hecho el depósito de ley: pp201602FA4721

ISSN-L: 2542-3029; ISSN: 2610-802X

Universidad Nacional Experimental Francisco de Miranda (UNEFM). Santa Ana de Coro. Venezuela

Eduardo Francisco García-Cabezas; Kevin Humberto Álvarez-Núñez; Jhonny Marcelo Orozco-Ramos; Juan Carlos Cayán Martínez

\section{Instalación de circuitos}

Para la instalación de circuitos se empleó un convertidor de energía que transforma de $12 \mathrm{~V}$ a $5 \mathrm{~V}$ ya que la raspberry funciona a $5 \mathrm{~V}$, ya a fuente de $12 \mathrm{~V}$ es utilizado para alimentar de energía a los motores de las ruedas. Se requiere de un "puente H" que controla el giro del motor de horario y anti horario como se muestra en la figura 9.

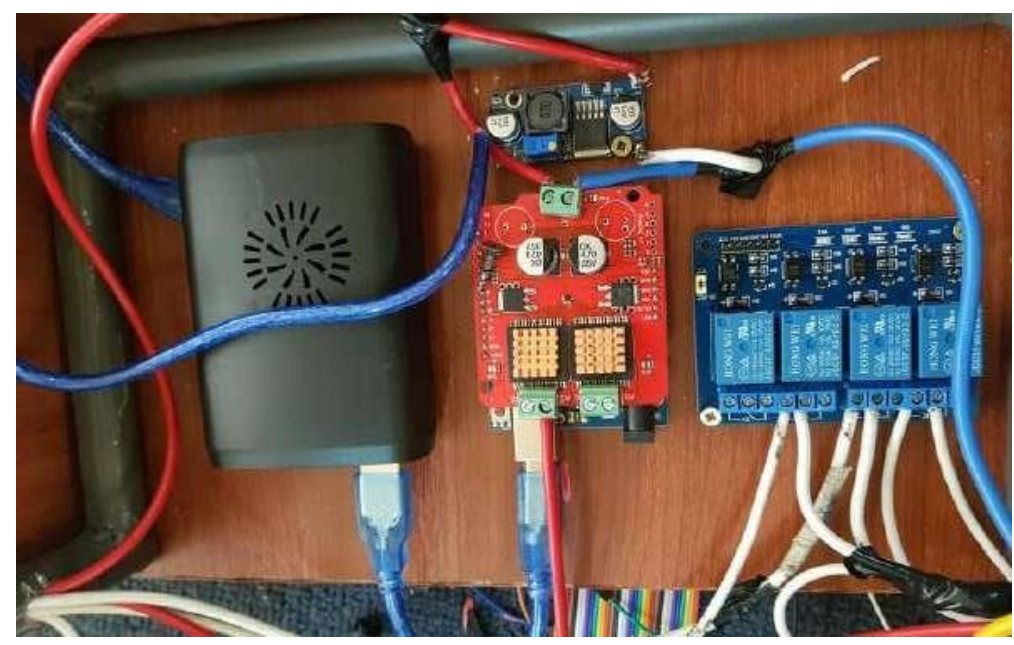

Figura 10. Instalación de circuitos.

Fuente: Los autores.

La figura 10. Muestra el puente $\mathrm{H}$ colocado sobre del Arduino según los pines seleccionados para la programación establecida, las salidas de los módulos de relé conectados a la electroválvula y a los motores de las ruedas. La raspberry conectada al Arduino y la pantalla touch por medio del cable HDMI. 


\section{CIENCIAMATRIA}

Revista Interdisciplinaria de Humanidades, Educación, Ciencia y Tecnología

Año VII. Vol. VII. N¹. Edición Especial. 2021

Hecho el depósito de ley: pp201602FA4721

ISSN-L: 2542-3029; ISSN: 2610-802X

Universidad Nacional Experimental Francisco de Miranda (UNEFM). Santa Ana de Coro. Venezuela

Eduardo Francisco García-Cabezas; Kevin Humberto Álvarez-Núñez; Jhonny Marcelo Orozco-Ramos; Juan Carlos Cayán Martínez

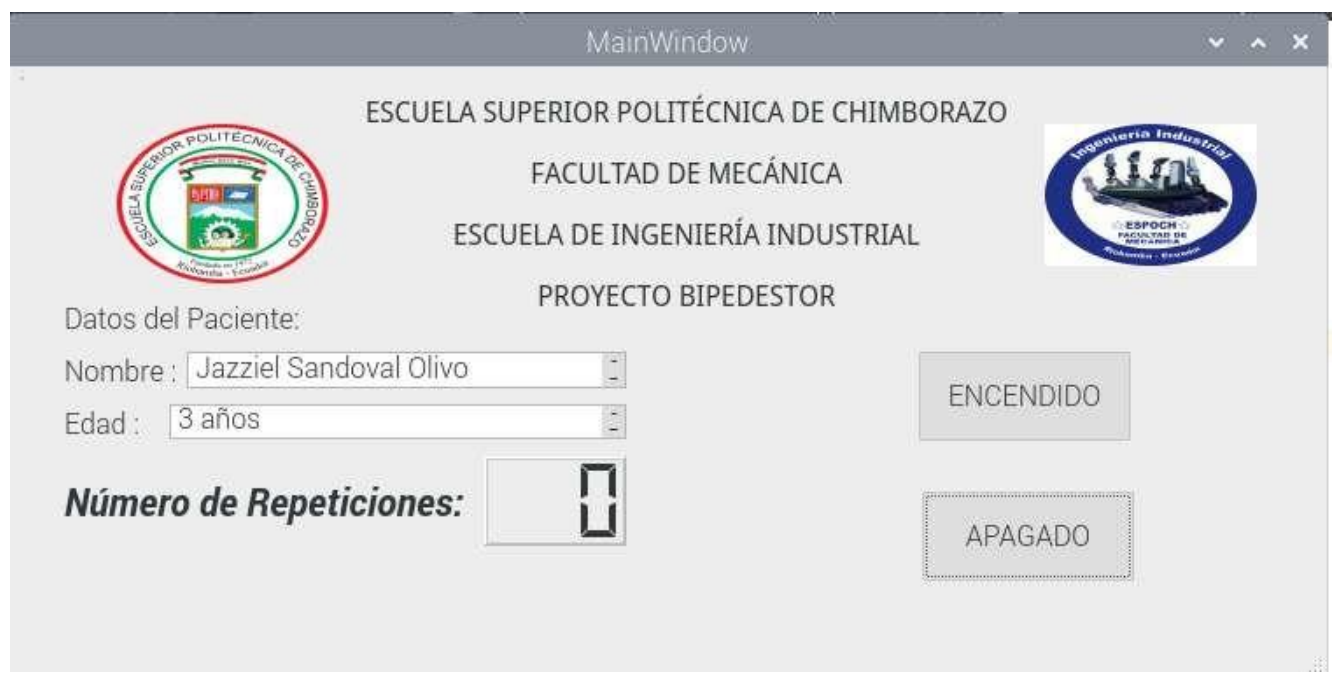

Figura 11. Interfaz gráfica.

Fuente: Los autores.

Para la configuración de la pantalla touch se utilizó en primera instancia un monitor con HDMI para poder instalar el sistema operativo y proceder a instalar desde la página oficial del fabricante waveshare el driver de la pantalla touch 5 pulgadas HDMI. Luego de instalar los drivers se descarga la carpeta que contendrá el archivo a ejecutar que destinara hacia la pantalla HDMI con el comando "cd LCD-show/" luego de abrir la carpeta ejecutamos el archivo "./LCD5-show" y nos dirige la pantalla.

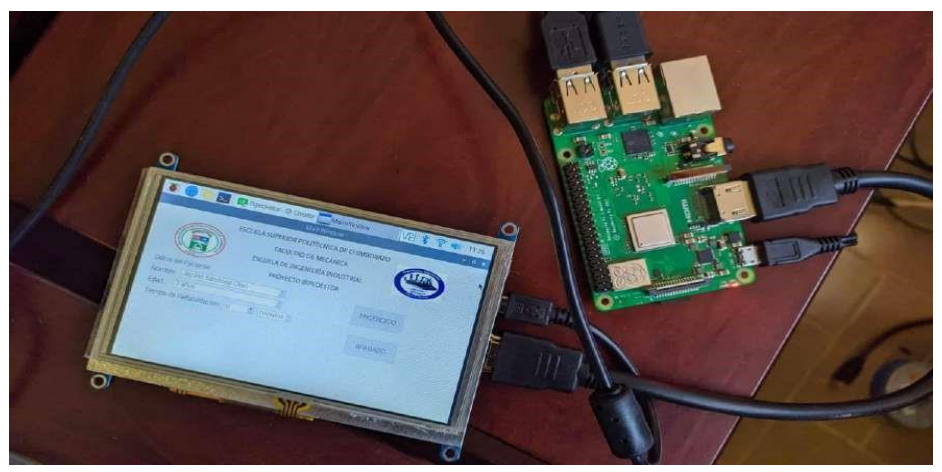

Figura 12. Conexión entre la Raspberry y la pantalla Touch. Fuente: Los autores. 


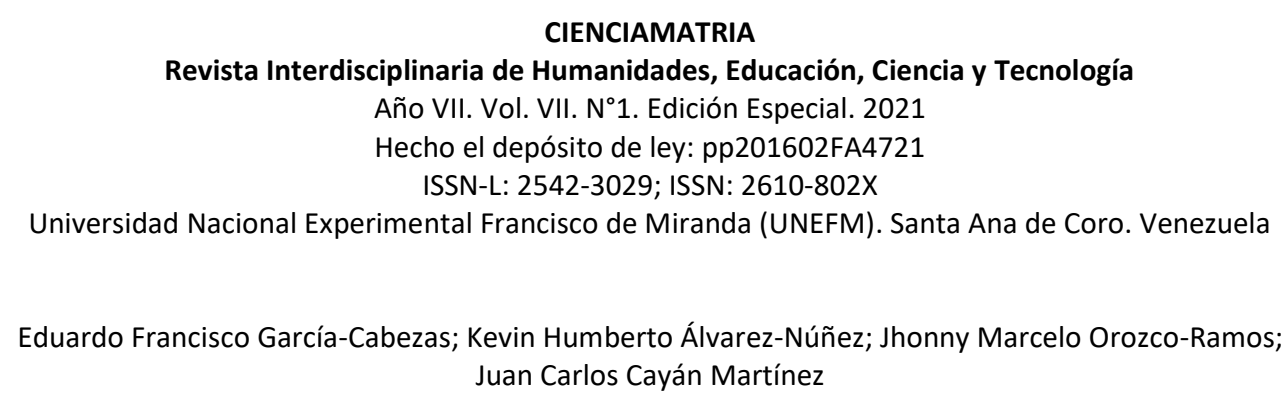

La interfaz gráfica en la pantalla touch muestra los datos del paciente, el tiempo de rehabilitación y el control de encendido y apagado del sistema. Dentro del botón de encendido al presionarlo envía una señal hacia el Arduino para encender todo el sistema del bipedestor y así controlar el cilindro neumático para ejercer la rehabilitación física y el movimiento de la máquina.

\section{CONCLUSIONES}

Mediante la presente investigación se logró desarrollar un bipedestor, diseñando e implementando un diseño mecánico automatizado para personas parapléjicas de 2 a 3 años, cumpliendo con mantener al niño en una posición bípeda y generando rehabilitaciones en sus extremidades inferiores.

Se analizó los parámetros de medidas antropométricas tomadas de niños de 2 a 3 años de Ecuador y por medio de software de diseño SolidWork se analizó las especificaciones de resistencia mecánica, facilidad de movimiento, transporte, mantenimiento. Cumpliendo con los ángulos de seguridad correspondientes para los estados de sedestación y bipedestación.

Los análisis estáticos son consistentes con el modelo construido y sus propiedades mecánicas de los materiales son óptimas generando seguridad siendo un factor importante en el proceso de la terapia física constantes.

Se implementó un sistema de control automatizado para el bipedestor con el uso de software y hardware libres, que por medio de una interfaz gráfica permite la comunicación entre los elementos gráficos y los usuarios controlando, monitoreando el bipedestor desarrollad.

Al utilizar el bipedestor no abrir las cajas donde se encuentran las instalaciones eléctricas y neumáticas para evitar accidentes tanto del usuario como de la máquina y manejar con cuidado el joystick. 
Es obligatorio utilizar el arnés de seguridad acoplado en las piernas cada vez que se inicie la rehabilitación y disponer del acompañamiento de personas adultas que se encuentren en capacidades de brindar ayuda a los niños.

Es necesario quitar cualquier obstáculo que se encuentre en el piso para evitar volcamientos de la máquina.

\section{AGRADECIMIENTO}

Agradecemos a los colaboradores indirectos de esta investigación, que prestaron las áreas del Laboratorio AQLAB y al Laboratorio de la Faculta de Ciencias, Sede de Orellana de la Escuela Superior Politécnica de Chimborazo.

\section{REFERENCIAS}

Arias, F. (2006) El Proyecto de Investigación introducción a la metodología científica. 5ta. Edición. Editorial Episteme, C.A. Caracas. Venezuela.

Bermeo, A. (2012). Ayudas para la marcha en la páralisis cerebral infantil. Revista internaional de Ciencias Podológicas. VI(1), pp. 9-24.

Catañeda, I.( 2014). La Red de Género y Salud ALAMES-Cuba como mecanismo de apoyo del Sistema Nacional de Salud. Revista Cubana de Salud Pública. 40(1).

Criollo, A. \& David, L. (2007). Diseño e implementación de un módulo electromecánico para la rehabilitación física de la mano derecha afectada como secuela de un ataque cerebrovascular. Universidad y Salud, II(14), pp. 60-61.

Galindo \& Gloria. (1997).Rehabilitación profesional y oportunidad laboral para el discapacitado en Costa Rica. Medicina Legal de Costa Rica, 13(2).

Luengas, L. \& Esperanza, C. (2013). Estudio de los rangos articulares en la bipedestación estática en personas normlaes vs amputados transtibiales. Tecnura, 17(2).pp. 61-63.

Meneses, C. \& Peñaloza, Y. (2015). Aplicación de la terapia robótica para el tratamiento de la mano espastica del adulto con hemiplejía. Revista Mexicana de Medicina Física y Rehabilitación, 27(3), p. 81. 
Murguía, A. \& Hernández, R. (2013). Factores de riesgo de trauma obstétrico. Ginecol Obstet, 81(6), pp. 297-303.

Palella, S. Y Martins, F. (2010) Metodología de la investigación cuantitativa. 3ra edición. Editorial FEDUPEL. Caracas.

Quiñones, K. \& Robayo, A. (2013). Diseño de un prototipo de bipedestador para pacientes pediátricos con espina bífida. Revista de la Facultad de Medicina 61(4), 2013. pp. 423-429.

Ruiz, E. \& Inche, J.(2008). Desarrollo de una interfaz hombre máquina orientada al control de procesos. Industrial Data, 11(1), pp. 70-72.

Sáenz, F. \& Chacón, E. (2014). Desarrollo de una interfáz para el aprendizaje y reconocimiento automático del lenguaje de signos. Revista lberoamericana de Educación en Tecnología y Tecnología en Educación, Issue 13, pp. 18-26.

Saavedra, R. \& García, E. (2013). Diseño de un equipo de bipedestación. Revista Ingenieria UC, 20(1), pp. 25-33. 